\title{
Combining laser microdissection and microRNA expression profiling to unmask microRNA signatures in complex tissues
}

\author{
Susanna Skalicky ${ }^{\ddagger 1}$, Peter J Zwiers ${ }^{\ddagger, 2}$, Timara Kuiper², Elisabeth Schraml', Matthias Hack|*,1 \& Grietje Molema,3
}

\section{ABSTRACT}

Neglecting tissue heterogeneity during the analysis of microRNA (miRNA) levels results in average signals from an unknown mixture of different cell types that are difficult to interpret. Here we demonstrate the technical requirements needed to obtain high-quality, quantitative miRNA expression information from tumor tissue compartments obtained by laser microdissection (LMD). Furthermore, we show the significance of disentangling tumor tissue heterogeneity by applying the newly developed protocols for combining LMD of tumor tissue compartments with RT-qPCR analysis to reveal compartmentspecific miRNA expression signatures. An important advantage of this strategy is that the miRNA signature can be directly linked to histopathology. In summary, combining LMD and RT-qPCR is a powerful approach for spatial miRNA expression analysis in complex tissues, enabling discovery of disease mechanisms, biomarkers and drug candidates.

\section{METHOD SUMMARY}

Laser microdissection was used on snap-frozen and cryostat-cut human tumor tissues to isolate tumor cells, stromal cells and tumor microvascular compartments. Total RNA was extracted from these compartments and used for highly parallelized microRNA (miRNA) quantification in primer-coated 384-well plates for high-throughput characterization of miRNA transcription. Compartment-specific miRNA signatures were thus revealed, which creates novel insights into the molecular status of different cell types that are located within the complex, heterogenic tumor tissue.

\section{KEYWORDS}

biomarker discovery • drug discovery • laser microdissection (LMD) $\cdot$ microRNA profiling $\cdot$ tissue complexity $\cdot$ tumor microenvironment

'TAmiRNA GmbH, Leberstraße 20, 1110 Vienna, Austria; ${ }^{2}$ Dept. Pathology \& Medical Biology, Medical Biology Section, University Medical Center Groningen, University of Groningen, Hanzeplein 1, 9713 GZ, Groningen, The Netherlands; ${ }^{3}$ Vivomicx BV, H.W. Mesdagstraat 53, 9718 HD, Groningen, The Netherlands; *Author for correspondence: office@tamirna.com; tThese authors contributed equally to this work

BioTechniques 67: 276-285 (December 2019) 10.2144/ btn-2019-0032
Laser microdissection (LMD) was developed in 1996 at the National Cancer Institute [1]. It is an automated sample-preparation technique that enables isolation of specific cells from a mixed population under microscopic visualization. This technique is characterized by the use of a laser coupled to an inverted light microscope to selectively extract a defined cell population of interest from a heterogeneous mixture of cells in a typical tissue section [2]. LMD allows us to combine histopathological information obtained by a dedicated pathologist with unmasking the molecular signatures associated with the histopathological findings. There is no need to enzymatically disrupt the tissue to obtain single cells, which results in no disturbance in the biomolecule make-up or activation status of the cells under study [3].

microRNAs (miRNAs) are short, noncoding RNAs that act as important post-transcriptional regulators of gene expression, including many key regulatory proteins involved in cancer development and progression. They can inhibit or enhance the expression of oncogenes or tumor suppressor genes, therefore profoundly affecting the biology of cancer [4]. The transcriptional regulation of miRNAs is mediated by RNA polymerase II promoters for intergenic miRNAs, or depends on the host gene in case of intronic miRNAs. Thus, miRNA transcription is a dynamic and tightly regulated process, and as a consequence every tissue exhibits a highly specific miRNA pattern that distinguishes it from other tissue types [5]. These patterns are highly conserved across species [6], and eventually are the origin of distinct extracellular miRNA profiles in cell-free biofluids such as serum and plasma [7]. This knowledge generated in recent decades now provides the basis for using circulating miRNAs as specific biomarkers for diagnosis of organ injury due to drug toxicity [8], or for the application as tumor biomarkers for the identification of cancer tissue origins [9]

However, one of the challenges that need to be solved in order to make a rational choice for miRNAs as biomarker or drug targets is posed by exactly this intrinsic heterogeneity in miRNA expression between different cell types and intrinsic cellular complexity of an organ or tissue. Here, we describe a novel protocol that consists of the combination of LMD with a highly sensitive PCR-based quantification method for miRNAs. The protocol allows us to increase the resolution of assigning production of miRNAs to cell subsets within a complex tissue, including tumors, and to disentangle a complex profile of signals caused by heterogeneity of cell types that are found within such tissues.

\section{MATERIALS \& METHODS}

Human tumor tissues

One pancreas ductal adenocarcinoma (PDAC) and two colorectal adenocarcinomas (CRCs) were commercially obtained from Indivumed (Indivumed $\mathrm{GmbH}$, Hamburg, Germany; Indivumed acts in strict compliance with the Declaration of Helsinki and the Convention for the Protection of Human Rights and Dignity of the Human Being with regard to the Application of Biology and Medicine: Convention on 


\begin{tabular}{|l|l|l|}
\hline Table 1. Primer sequences used for mRNA qRT-PCR analysis. \\
\hline Gene & Forward primer & Reverse primer \\
\hline GAPDH & TACACTGAGGACCAGGTTGTG & TTGACGAAGTGGTCGTTGAG \\
\hline ACTB & CACGCCATCCTGCGTCTGGA & AGCACCGTGTTGGCGTAGAG \\
\hline
\end{tabular}

- Human Rights and Biomedicine). A dedicated pathologist histologically assessed hematoxylin-stained cryostat-cut tumor sections and judged them as PDAC and $\mathrm{CRC}$, and assisted in annotating tumor cell and stromal areas for further processing and handling in the LMD procedure.

\section{Laser microdissection}

$9 \mu \mathrm{m}$ cryostat-cut tumor sections were used to laser microdissect tumor tissue (TT), stromal tissue (ST) and tumor endothelial cells (TECs). For this purpose, the cryosections were placed on polyethylene naphthalate (PEN) membrane slides (Carl Zeiss B.V., Breda, The Netherlands) and stained with hematoxylin to visualize tumor tissue histology. For visualization of TEC, cryosections were placed on PET-Frame Slides (Leica Microsystems, Wetzlar, Germany) and stained with FITClabeled Ulex Europaeus Agglutinin (UEA I, Brunschwig Chemie, Amsterdam, The Netherlands). Tissue regions of interest were selected using LMD6500 software v7.0, (Leica Microsystems) and total areas varying from $1 \times 10^{4}$ to $2 \times 10^{6} \mu \mathrm{m}^{2}$ were microdissected using the LMD6500 system (Leica Microsystems). Laser microdissected material was collected in a $0.5-\mu \mathrm{l}$ adhesive cap (Carl Zeiss B.V.) and stored at $-80^{\circ} \mathrm{C}$ until further processing.

\section{Total RNA extraction}

RNA extraction was performed using the miRNeasy Micro kit (Qiagen, Germany). Prior to extraction, $1 \mu \mathrm{l}$ of a synthetic RNA mix (Qiagen, Germany) consisting of three different synthetic control RNAs (UniSp2, $2 \mathrm{fmol} / \mathrm{ml}$; UniSp4, $0.02 \mathrm{fmol} / \mathrm{ml}$; UniSp5, $0.0002 \mathrm{fmol} / \mathrm{ml}$ ) was added to $700 \mu \mathrm{l}$ QIAzol For sample lysis, $350 \mu \mathrm{I}$ QIAzol was added in two steps to the tissue and vortexed carefully for 20 s inbetween. Lysates were centrifuged at low speed and transferred to a $1.5-\mathrm{ml}$ Eppendorf tube. The samples were homogenized by vortexing for $1 \mathrm{~min}$ and placed at room temperature $\left(15-25^{\circ} \mathrm{C}\right)$ for $5 \mathrm{~min} .140 \mu \mathrm{l}$ chloroform was added followed by vigorous mixing and incubation at room temperature for 2-3 min. Samples were centrifuged for 15 min at $12,000 \times g$ at $4^{\circ} \mathrm{C}$. The upper aqueous phase was transferred to a new tube, precipitated and purified on a QIAcube liquidhandling robot using the miRNeasy Micro kit (Qiagen, Hilden, Germany) according to the manufacturer instructions, with the following modification: glycogen (Ambion, TX, USA) was added to the aqueous phase to a final concentration of $50 \mu \mathrm{g} / \mathrm{ml}$. RNA was eluted in a single round in $30-\mu$ l nuclease-free water and stored at $80^{\circ} \mathrm{C}$. Total RNA yields from PDAC tumor compartments were assessed using the Agilent Bioanalyzer 2100 platform (Supplementary Figure S1) together with the RNA 6000 Pico kit (Agilent Technologies, CA, USA) and used for correlation against reference gene expression by Pearson correlation.

\section{miRNA RT-qPCR quantification}

Equal volumes of total RNA extracts from every sample were used directly for reverse transcription into cDNA using the miRCURY LNA RT Kit (Qiagen, Hilden, Germany). Universal reverse transcription reactions for RT-qPCR screening were performed as either 10 - or $45-\mu$ reactions containing 4or $18-\mu$ l total RNA input, respectively. A synthetic cDNA spike-in (cel-miR-39-3p, $0.002 \mathrm{fmol} / \mu \mathrm{l}$ ) was added to the RT mix to monitor variance in RT efficiency as well as to detect the presence of enzyme inhibitors. For qPCR analysis, cDNA samples were diluted 1:100. qPCR reactions were performed as $10-\mu$ l reactions in 96 -well plates for analysis of individual miRNAs. For screening purposes, 384-well plates (Human Panel I V4, Exiqon, Denmark) were used, which contained 375 miRNA-specific primers, four spike-in primers (UniSp2, UniSp4, UniSp5 and cel-miR-39-3p), three distinct PCR control wells with precoated DNA templates and primers referred to as 'interplate-control 3' (IPC3) and one negative control well. All primers were obtained from Qiagen and use locked nucleic acid (LNA) technology for optimi- zation of hybridization efficiencies. For PCR the miRCURY LNA SYBR Green PCR mix was used (Qiagen, Hilden, Germany) and plates were prepared using an Eppendorf epMotion liquid-handling robot. PCR amplification was conducted in a Roche LC480 II instrument (Roche, Hildesheim, Germany) with the following settings: $95^{\circ} \mathrm{C}$ for $10 \mathrm{~min}, 45$ cycles of $95^{\circ} \mathrm{C}$ for $10 \mathrm{~s}$ and $60^{\circ} \mathrm{C}$ for $60 \mathrm{~s}$, followed by melting curve analysis. $\mathrm{Cq}$ values were computed using the second derivative maximum method provided with the LC480 II software.

For targeted miRNA analysis, $\mathrm{Cq}$ values were normalized using the delta-Cq $(\mathrm{dCq})$ method to the global mean of all RNA spike-in controls (UniSp2, UniSp4, UniSp5) to account for any technical variation introduced during the analysis. Other small noncoding RNAs, specifically 5 S rRNA, U6 snRNA and U48 snRNA, were assessed to account for differences in total RNA content/cell number between the tissue compartments. In order to account for differences in total RNA content in LMD samples due to differences in cell numbers or compartment sizes (especially relevant for whole tumor), global mean normalization was performed [10].

\section{mRNA RT-qPCR quantification}

For mRNA quantification the TATAA SYBR Grandmaster mix kit (TATAA Biocenter, Sweden) was used. $8 \mu$ lof total RNA extracts were used for reverse transcription and all steps were carried out according to recommendations by the manufacturer. PCR amplification was performed in a 96-well format in a Roche LC480 II instrument (Roche, Germany) with the following settings: $95^{\circ} \mathrm{C}$ for $30 \mathrm{~s}$ followed by 45 cycles of $95^{\circ} \mathrm{C}$ for $5 \mathrm{~s}$, $63^{\circ} \mathrm{C}$ for $15 \mathrm{~s}$ and $72^{\circ} \mathrm{C}$ for $10 \mathrm{~s}$, and subsequent melting curve analysis (data not shown). To calculate the cycle of quantification values (Cq-values), the second derivative method was used. Primer sequences used for GAPDH and ACTB are listed in Table 1. 


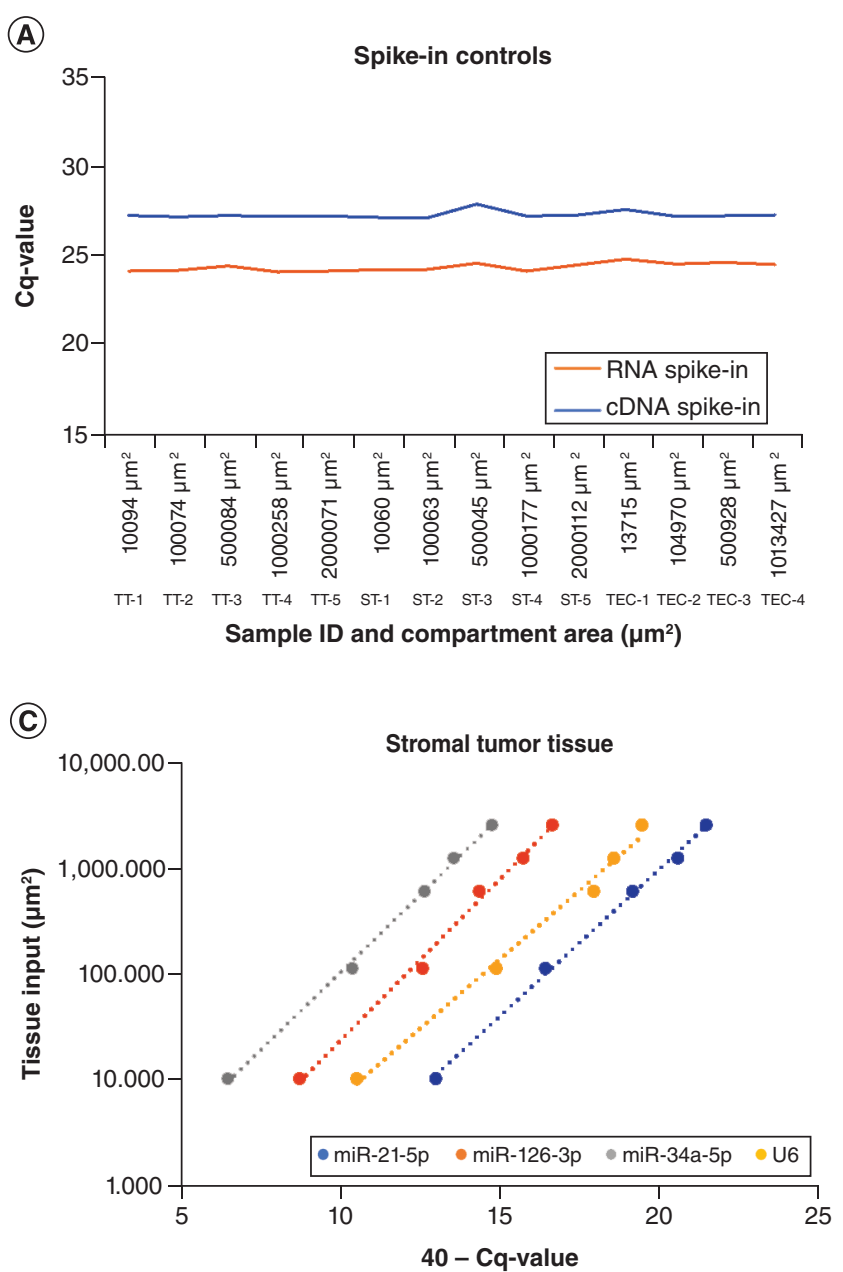

(E)

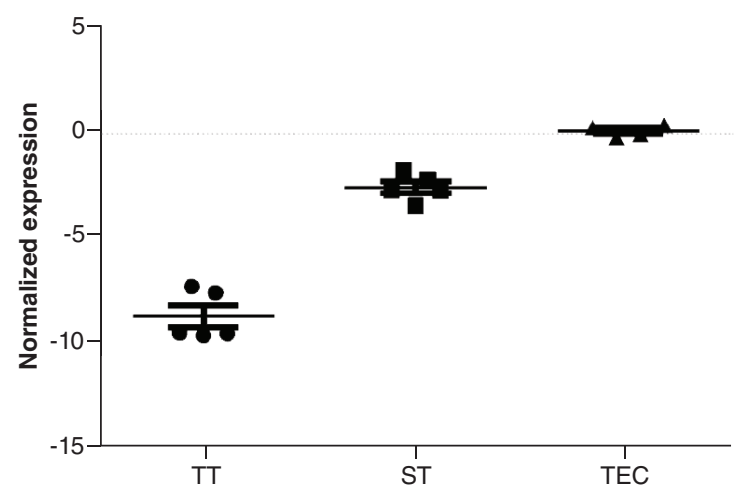

(B)

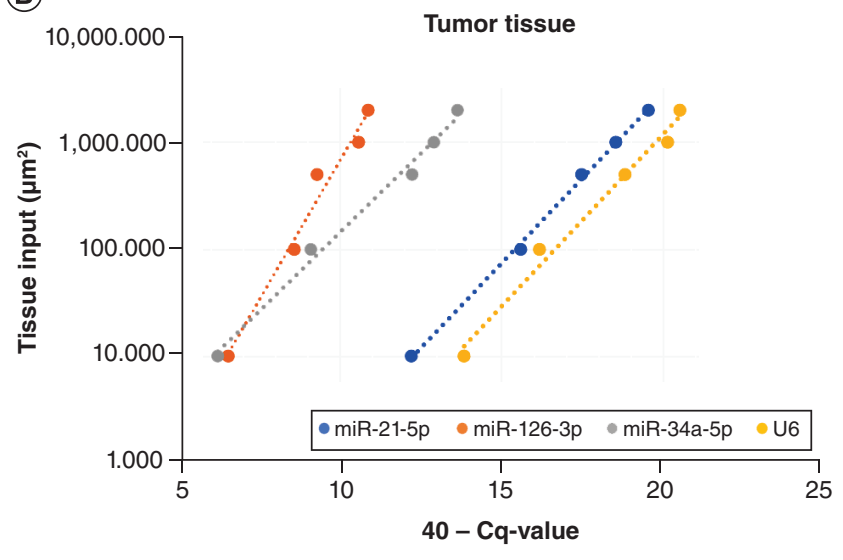

(D)

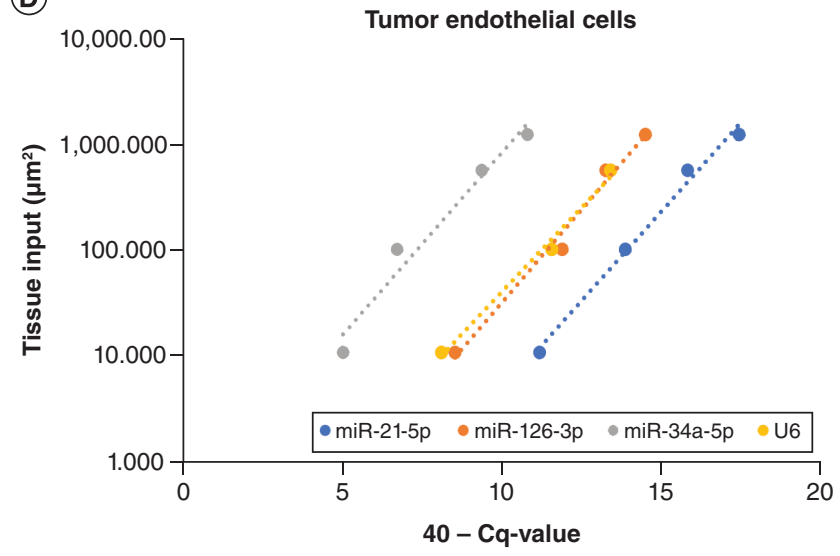

miR-126-3p

Figure 1. Linearity between laser microdissected tissue compartment area and miRNA quantification enables downscaling of sample input obtained by laser microdissection to areas of $10,000 \mu \mathrm{m}^{2} .9 \mu \mathrm{m}$ cryostat-cut colorectal carcinoma tumor tissue sections were subjected to laser microdissection to yield TT, ST and TEC at different surface areas (see also 'Materials \& methods' section for information). Targeted RT-qPCR was used for quantification of two spike-in controls, which were either added prior to RNA extraction (RNA spike-in UniSp4) or during cDNA synthesis (cDNA spike-in [cel-miR-39]), three miRNAs (miR-21-5p, miR-126-3p and miR-34a-5p), as well as one reference noncoding RNA (U6 snRNA) in total RNA extracts derived from tissue compartments of varying size $\left(1 \times 10^{4}-2 \times 10^{6} \mu \mathrm{m}^{2}\right)$. (A) Spike-in controls show that RNA isolation and reverse transcription efficiency are independent of the compartment area size. (B-D) A strictly linear relation between laser microdissection area and qPCR signal was found in all tumor compartments. (E) miR-126-3p expression normalized to laser microdissected $\mu \mathrm{m}^{2}$ input in the RT-qPCR analysis. Each dot represents a compartment area between $1 \times 10^{4}$ and $2 \times 10^{6} \mu \mathrm{m}^{2}$. Differences in miR-126-3p levels between compartments can be detected irrespective the compartments.

ST: Stromal tissue; TEC: Tumor microvasculature; TT: Tumor tissue. 

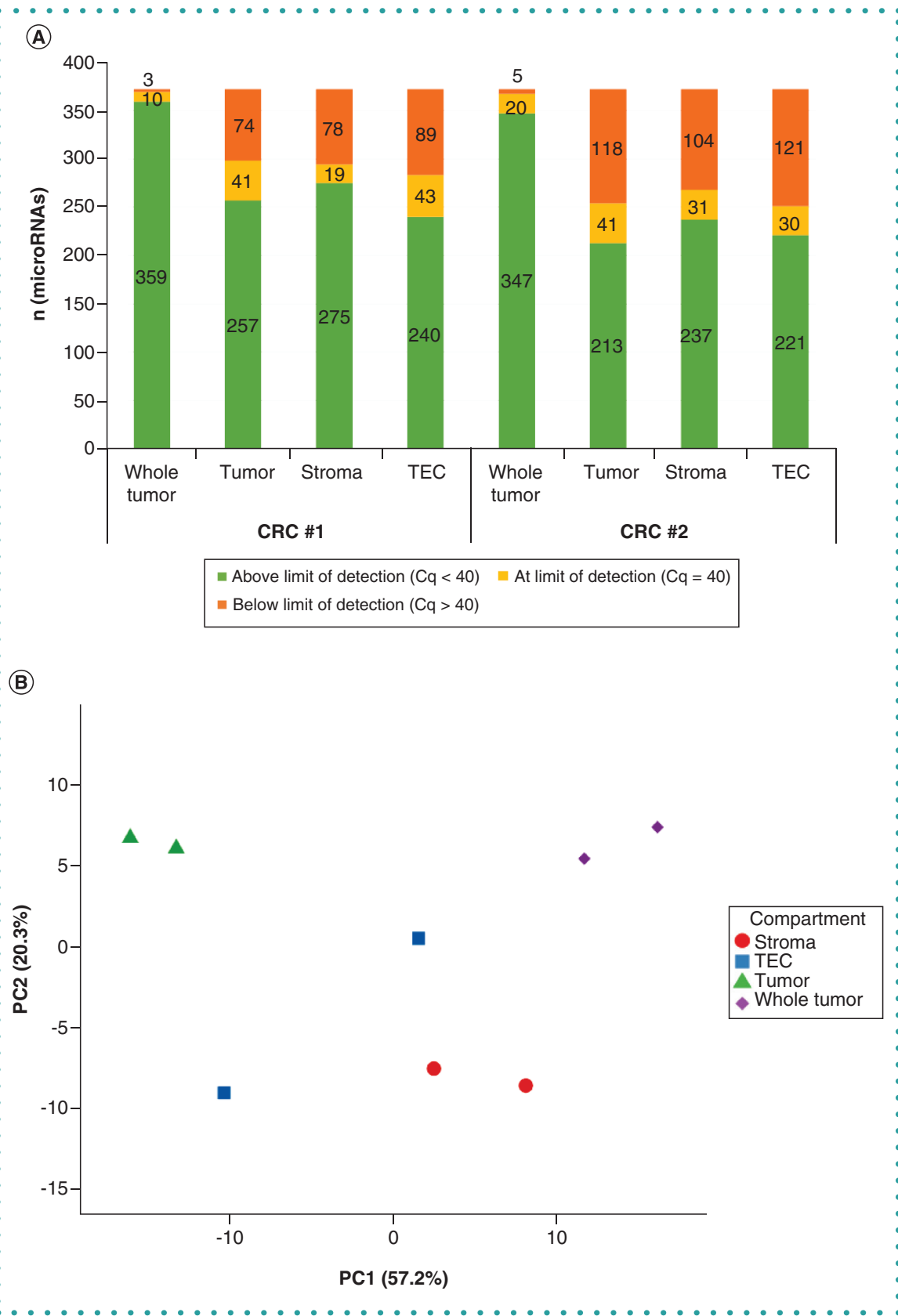

Figure 2. miRNA screening and clustering in tissue compartments. (A) Total RNA extracted from two colorectal carcinoma samples (whole tumor) as well as the respective tumor cell, tumor stromal cell and TEC compartments was used for screening of 384 distinct miRNA species. On average, more than 250 miRNAs were detected in tissue compartments above the limit of detection. (B) The principal component analysis conducted on the basis of 255 miRNAs that were detected across all compartments as well as whole tumor. PC1 explains $57.2 \%$ of the overall variance in miRNA levels, while PC2 explains $20.3 \%$. The relative positioning of samples in the plot provides information about their similarity in terms of microRNA expression.

PC1: Principal component 1; PC2: Principal component 2; TEC: Tumor endothelial cell.

\section{- Statistical analysis}

Principal component analysis (PCA) was performed using Clustvis [11] to perform unsupervised clustering. Medcalc was used for Pearson correlation analysis between total RNA concentration and reference gene levels.

\section{RESULTS \& DISCUSSION}

RT-qPCR can be applied for miRNA quantification in LMD tumor tissue compartments

One of the main challenges associated with RNA quantification in LMD tissue compartments that measure as low as $10,000 \mu \mathrm{m}^{2}$ surface with 9-10- $\mu \mathrm{m}$ thickness is the low total RNA yield, which generally ranges between 10 and $500 \mathrm{pg} / \mu \mathrm{l}$. This is well below the limit of detection for photometric quantification methods such as Nanodrop, and potentially challenging to quantify using fluorescence-based methods. Therefore, we initially investigated the feasibility of miRNA quantification using an RT-qPCR platform on the basis of samples from human CRC tissue. This tissue was compartmentalized by LMD into TT, ST and tumor microvasculature (mainly consisting of tumor endothelial cells [TECs]). For every compartment LMD replicates with areas varying between $10^{4} \mu \mathrm{m}^{2}$ and $2 \times 10^{6} \mu \mathrm{m}^{2}$ were generated at Vivomicx. miRNA quantification was performed at TAmiRNA: one synthetic spike-in control (RNA spike-In UniSp4) was added prior to RNA extraction, and one spike-in (cDNA spike-in cel-miR-39) was added to the total RNA in order to monitor the efficiencies of RNA extraction and reverse transcription, respectively. Both spike-ins, three endogenous miRNAs (miR-126-3p, miR-21-5p, miR-34a-5p) as well as one reference RNA (U6 snRNA) were analyzed by RT-qPCR.

The results obtained for RNA and cDNA spike-in controls showed that RNA extraction and RT efficiencies were comparable across all samples and independent of the tissue/RNA input amounts (Figure 1A). In all three compartments a strict linearity between tissue input and RT-qPCR signals was observed for endogenous miRNAs and U6 snRNA (Figure 1B-D). When focusing on miR-126-3p expression, a miRNA previously shown to be enriched in endothelial cells [12,13], this linearity between input of material in $\mu \mathrm{m}^{2}$ and signal in the RT-qPCR analysis was confirmed. The observed difference in miR-126-3p expression between tumor cells, stromal cells and tumor microvasculature was not dependent on the input but on the compartment under investigation (Figure 1E).

From this we concluded that differences in miRNA expression between the compartments can be reliably detected independent of the compartment size. This is an important conclusion, because the smaller the laser-microdissected compartments, the lower the risk of cross-contamination with other cell types, and the shorter the time required for the LMD procedure. 
Table 2. Top 20 tumor compartment-enriched microRNAs.

\begin{tabular}{|c|c|c|c|c|c|c|c|c|}
\hline & \multirow{2}{*}{$\begin{array}{l}\text { Tumor-enriched } \\
\text { microRNAs }\end{array}$} & \multicolumn{4}{|c|}{ Global mean normalized level (dCq) } & \multicolumn{3}{|c|}{ Enrichment factor ( $2^{\mathrm{ddCq}}$ ) } \\
\hline & & $\begin{array}{l}\text { Whole } \\
\text { tumor }\end{array}$ & Tumor (TT) & \begin{tabular}{|l} 
Stroma \\
(ST)
\end{tabular} & TEC & $\begin{array}{l}\text { TT vs } \\
\overline{\mathbf{X}}(\mathrm{ST}+\mathrm{TEC})\end{array}$ & \begin{tabular}{|l} 
ST vs \\
$\bar{X}(T T+T E C)$
\end{tabular} & \begin{tabular}{|l} 
TEC vs \\
$\bar{X}(T T+S T)$
\end{tabular} \\
\hline 1 & hsa-miR-182-5p & 1.67 & 2.46 & -3.62 & -1.11 & 28.26 & 0.05 & 0.69 \\
\hline 2 & hsa-miR-183-5p & 0.63 & 1.26 & -4.53 & -1.80 & 21.46 & 0.05 & 0.89 \\
\hline 3 & hsa-miR-375 & 2.45 & 3.41 & -0.90 & -0.82 & 19.34 & 0.22 & 0.24 \\
\hline 4 & hsa-miR-200b-3p & 6.89 & 7.51 & 1.72 & 4.76 & 19.24 & 0.05 & 1.10 \\
\hline 5 & hsa-miR-96-5p & 0.82 & 2.66 & -1.94 & -0.53 & 14.81 & 0.12 & 0.54 \\
\hline 6 & hsa-miR-200a-3p & 4.45 & 6.20 & 0.95 & 3.70 & 14.63 & 0.06 & 1.09 \\
\hline 7 & hsa-miR-135b-5p & 3.54 & 4.40 & -0.66 & 1.73 & 14.60 & 0.08 & 0.91 \\
\hline 8 & hsa-miR-194-5p & 6.64 & 7.49 & 2.54 & 4.93 & 13.48 & 0.08 & 0.94 \\
\hline 9 & hsa-miR-196a-5p & 1.35 & 2.31 & -2.57 & -0.30 & 13.41 & 0.08 & 0.89 \\
\hline 10 & hsa-miR-301b & -4.09 & -0.76 & -5.16 & -3.72 & 12.82 & 0.13 & 0.59 \\
\hline 11 & hsa-miR-147b & -5.65 & -3.24 & -7.57 & -6.20 & 12.52 & 0.14 & 0.58 \\
\hline 12 & hsa-miR-17-5p & 0.64 & 2.79 & -2.18 & 0.49 & 12.37 & 0.07 & 1.14 \\
\hline 13 & hsa-miR-429 & 3.35 & 4.74 & -0.08 & 2.35 & 12.15 & 0.08 & 1.01 \\
\hline 14 & hsa-miR-215 & 5.91 & 6.59 & 1.73 & 4.30 & 11.90 & 0.08 & 1.10 \\
\hline 15 & hsa-miR-584-5p & -3.69 & -1.68 & -6.18 & -4.20 & 11.40 & 0.11 & 0.83 \\
\hline 16 & hsa-miR-135a-5p & -0.22 & 0.64 & -4.04 & -1.71 & 11.38 & 0.09 & 0.99 \\
\hline 17 & hsa-miR-192-5p & 6.79 & 7.82 & 3.14 & 5.65 & 10.75 & 0.08 & 1.12 \\
\hline 18 & hsa-miR-141-3p & 7.11 & 8.21 & 3.82 & 5.92 & 10.15 & 0.11 & 0.93 \\
\hline 19 & hsa-miR-200c-3p & 7.88 & 8.46 & 3.98 & 6.31 & 9.92 & 0.09 & 1.06 \\
\hline 20 & hsa-miR-20a-5p & 6.60 & 8.59 & 4.60 & 6.14 & 9.31 & 0.15 & 0.73 \\
\hline
\end{tabular}

$\mathrm{dCq}$ values reflect the $\log _{2}$-transformed expression level. Positive values mean levels higher than the global mean (high abundant), while negative values mean levels below the global mean, that is, low abundance.

dCq: Delta-Cq; ST: Stromal tissue; TEC: Tumor endothelial cell; TT: Tumor tissue.

Normalization strategies for spatial miRNA profiling within or across tumor tissue compartments

In the next step, we aimed to address the issue of normalization to enable comparison of miRNA expression levels within one tissue compartment (e.g., tumor cells) between different tumor specimens. Normalization can be achieved by keeping the compartment areas constant across all samples. By monitoring RNA extraction efficiency using spike-in controls (Figure 1), the technical variability and quality of RNA isolation, reverse transcription and PCR amplification can be assessed. However, especially when comparing miRNA expression between different tissue compartments that are composed of different cell types, normalization to the size of the laser-microdissected

Vol. $67 \mid$ No. 6 | (๑) 2019 Matthias Hackl compartment will bias the results because the number of cells contained in a defined compartment area will be different depending on the type of cells. Consequently, normalization needs to be performed at either the total RNA level by using highly sensitive quantification methods capable of detecting picogram amounts of RNA, or by using reference RNAs. The latter are presumably transcribed at constant levels across various cell types and are therefore correlated to the amount of total RNA/number of cells in a specific tissue compartment.

We addressed this issue by compartmentalizing a PDAC sample into TT, ST and TEC (270,000 $\mu \mathrm{m}^{2}$ per compartment), and analyzing the levels of three non-coding RNA references (U6 snRNA, U48 snRNA and $5 S$ rRNA) and two coding RNA references
(GAPDH, ACTB), respectively. Cq-values obtained for five reference gene candidates across three tumor compartments (tumor, stroma and vasculature) were correlated to the log2-transformed total RNA concentration in each compartment, which was determined using the Agilent RNA 6000 Pico kit. We observed the highest correlation between U6 snRNA levels and total RNA (Pearson correlation coefficient [PCC] 0.98 ), but the other four reference RNAs also exhibited good correlation to total RNA (ACTB: 0.81; GAPDH: 0.80; U48: 0.86; 5S rRNA: 0.74). From these data, we concluded that the tested panel of reference RNAs and their respective geometric means are suitable for normalization of sample input variability introduced by differences in cell numbers in the laser-microdissected tissue compartments (TT, ST and TEC). 
Figure 3. Identification of compartment specific miRNA profiles. miRNA profiles were assessed in laser-microdissected colorectal carcinoma tissue compartments using 384-wel microRNA array platform technology. Fold changes in miRNA expression levels between compartments were calculated and plotted to highlight miRNAs with strong enrichment in the respective compartments. (A) Tumor-enriched miRNAs, (B) stroma-enriched miRNAs and (C) tumor microvasculature-enriched miRNAs. ST: Stromal tissue; TEC: Tumor microvasculature; TT: Tumor tissue.

Large-scale profiling of miRNA levels in human colorectal carcinoma tissue compartments identifies signatures of tumor, stroma \& microvasculatureenriched miRNAs

Next, we investigated whether the total RNA yield from tumor compartments was sufficient to conduct large-scale miRNA screening by RT-qPCR to enable unbiased discovery of miRNA signatures specific for tumor, stroma and microvasculature. LMD was performed on two $\mathrm{CRC}$ specimens from two different patients to obtain total RNA from the three compartments $\left(2.7 \times 10^{5} \mu \mathrm{m}^{2}\right)$, as well as a whole-tumor sections $(9 \mu \mathrm{m})$. In each sample the levels of 372 distinct miRNA species as well as 12 endogenous or synthetic control RNAs were analyzed using RT-qPCR in 384-well plates (see Supplementary File 1 for complete information on Cq-values in all samples). Figure $2 \mathrm{~A}$ depicts the number of miRNAs that were above the limit of quantification ( $\mathrm{Cq}<40$, green), at the limit of detection ( $\mathrm{Cq}=40$, yellow), as well as the number of miRNAs that were below the limit of detection $(\mathrm{Cq}>40)$. We observed the highest detection rates in whole-tumor specimens, 359 and 347 miRNAs above the LoQ, respectively, which is likely due to the higher RNA yield from whole-tumor sections compared with those from the laser-microdissected compartments (Figure 2A). Fewer miRNAs were detected in tumor compartments; however, 255 miRNAs were detected across all eight specimens. Unsupervised clustering analysis of whole tumor and tumor compartments was performed on the basis of this common set of 255 miRNAs using principal component analysis (PCA), even though this strategy excludes the fact

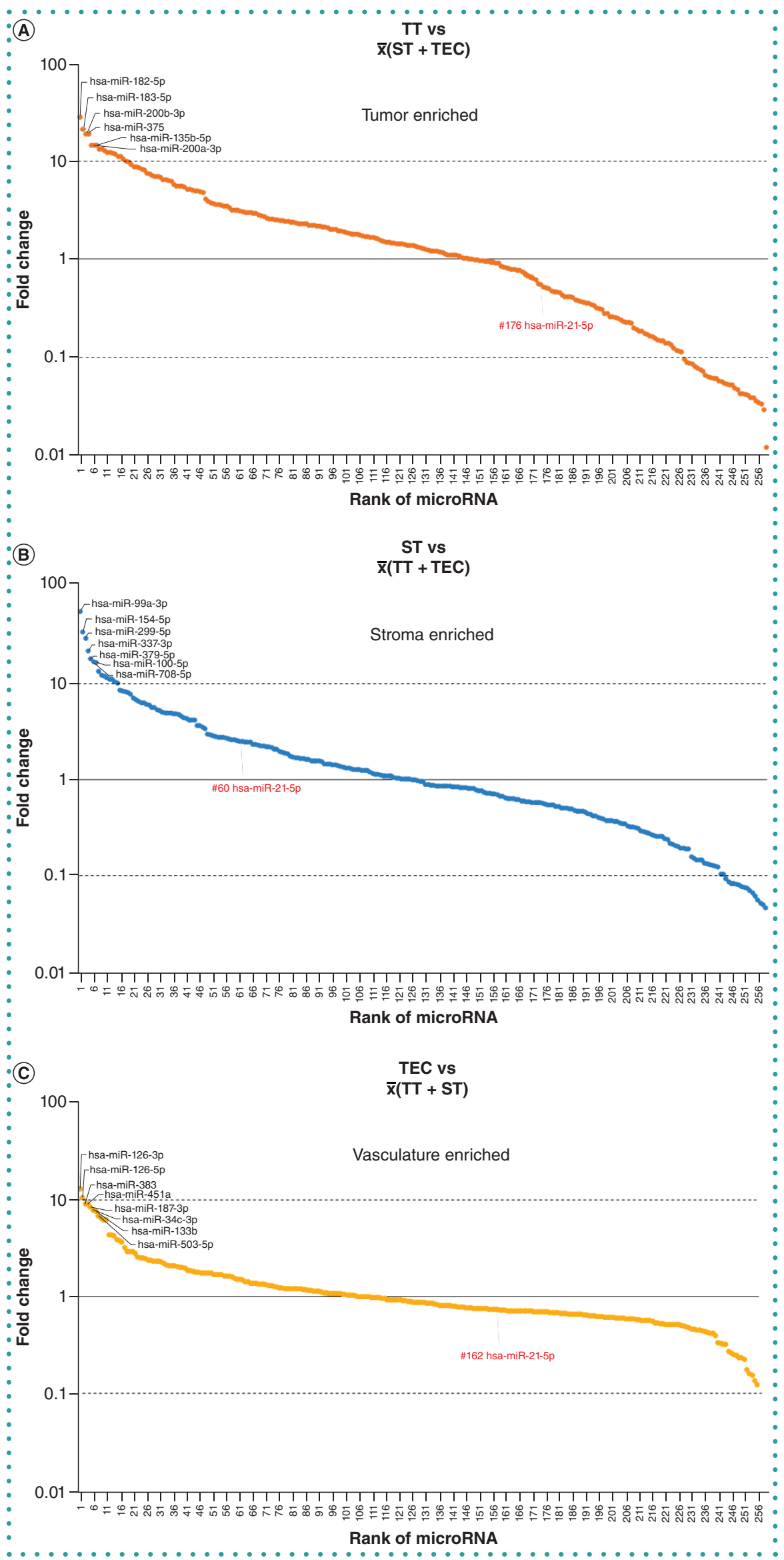




\begin{tabular}{|c|c|c|c|c|c|c|c|}
\hline \multirow{2}{*}{$\begin{array}{l}\text { Stroma-enriched } \\
\text { microRNAs }\end{array}$} & \multicolumn{4}{|c|}{ Global mean normalized level (dCq) } & \multicolumn{3}{|c|}{ Enrichment factor (2ddcq) } \\
\hline & Whole tumor & Tumor (TT) & Stroma (ST) & TEC & \begin{tabular}{|l} 
TT vs \\
$\bar{x}(S T+T E C)$
\end{tabular} & \begin{tabular}{|l} 
ST vs \\
$\bar{x}(T T+T E C)$
\end{tabular} & \begin{tabular}{|l} 
TEC vs \\
$\bar{x}(T T+S T)$
\end{tabular} \\
\hline hsa-miR-99a-3p & -3.67 & -7.48 & -1.00 & -6.20 & 0.07 & 57.26 & 0.26 \\
\hline hsa-miR-154-5p & -1.07 & -5.68 & 0.54 & -3.48 & 0.05 & 34.82 & 0.53 \\
\hline hsa-miR-299-5p & -3.42 & -7.05 & -1.27 & -5.32 & 0.07 & 30.31 & 0.45 \\
\hline hsa-miR-337-3p & -2.96 & -7.48 & -1.58 & -4.65 & 0.05 & 22.50 & 0.92 \\
\hline hsa-miR-379-5p & -1.74 & -7.48 & -0.46 & -1.82 & 0.01 & 18.34 & 4.43 \\
\hline hsa-miR-708-5p & 1.43 & -3.25 & 2.68 & 0.43 & 0.04 & 17.11 & 1.64 \\
\hline hsa-miR-100-5p & 3.89 & -0.55 & 5.28 & 2.96 & 0.04 & 16.85 & 1.51 \\
\hline hsa-miR-125b-5p & 6.22 & 2.16 & 7.70 & 5.71 & 0.04 & 13.59 & 1.71 \\
\hline hsa-miR-199b-5p & 2.14 & -0.42 & 4.48 & 2.09 & 0.08 & 12.53 & 1.04 \\
\hline hsa-miR-99a-5p & 2.68 & -0.90 & 3.83 & 1.34 & 0.09 & 12.20 & 0.92 \\
\hline hsa-miR-376a-3p & -2.42 & -6.19 & -1.05 & -2.99 & 0.06 & 11.65 & 1.55 \\
\hline hsa-miR-199a-3p & 4.52 & 0.01 & 5.51 & 4.02 & 0.04 & 11.33 & 2.39 \\
\hline hsa-miR-199a-5p & 2.58 & -0.66 & 4.69 & 3.04 & 0.04 & 11.33 & 2.03 \\
\hline hsa-miR-411-5p & -1.97 & -4.59 & -0.71 & -3.68 & 0.19 & 10.77 & 0.49 \\
\hline hsa-miR-214-3p & 1.75 & -1.41 & 3.64 & 1.94 & 0.05 & 10.39 & 1.77 \\
\hline hsa-miR-145-5p & 6.24 & 2.91 & 8.09 & 7.03 & 0.04 & 8.74 & 2.88 \\
\hline hsa-miR-377-3p & -5.75 & -7.48 & -3.55 & -5.79 & 0.14 & 8.51 & 0.83 \\
\hline hsa-miR-342-3p & 3.05 & 0.05 & 4.32 & 2.48 & 0.10 & 8.31 & 1.22 \\
\hline hsa-miR-370 & -4.16 & -7.48 & -2.73 & -4.05 & 0.06 & 8.19 & 2.08 \\
\hline hsa-miR-138-5p & -4.49 & -7.48 & -3.43 & -5.32 & 0.12 & 7.85 & 1.10 \\
\hline \multicolumn{8}{|c|}{$\begin{array}{l}d C q \text { values reflect the } \log _{2} \text {-transformed expression level. Positive values mean levels higher than the global mean (high abundant), while negative } \\
\text { values mean levels below the global mean, that is, low abundance. }\end{array}$} \\
\hline
\end{tabular}

that in one particular compartment expression of some miRNAs may be completely lacking. $\mathrm{PC} 1$ and $\mathrm{PC} 2$ were found to explain $77.5 \%$ of the overall variance in miRNA levels (Figure 2B). Clustering of samples in the PCA plot showed high similarity (short distance) between the two biological replicates of tumor compartments, while large differences (large distances) were observed between the compartments as well as whole tumor. This suggests the existence of compartmentspecific miRNA signatures.

In order to identify tumor, stroma and tumor microvascular signatures, pair-wise comparison of normalized miRNA levels between compartments was performed (Figure 3A-C). Figure 3A exemplifies several tumor-enriched miRNAs such as miR-17-5p,
miR-182-5p and miR-200b-3p, which have all been previously shown to be drivers of oncogenic phenotypes [14-16]. On the other hand, miR126-3p, which is known to be more highly expressed by endothelial cells [17-19], was found to be most abundant in the tumor microvasculature compartment alongside miR-133b. The latter is known to be enriched in muscle tissue, including vascular smooth muscle cells (VSMCs) [20], and may possibly be upregulated by either pericytes or endothelial cells that make up the tumor microvasculature by conditions that prevail in the tumor microenvironment. miRNAs that were found most enriched in stroma were miR-99a-3p and miR-154-5p, which are both known to be promoters of epithelial-to-mesenchymal transition and highly abundant in fibroblasts $[21,22]$.
Interestingly, miR-21-5p, which has been acknowledged several times as a miRNA eliciting oncogenic effects, as well as being associated with poor survival of CRC patients, was found to be expressed highest in the stroma compartment, followed by vasculature and tumor (Figure $3 \mathrm{~A}-\mathrm{C}$ ).

Extended lists of top 20 compartmentenriched miRNAs were generated based on fold enrichment in the target compartment compared with the average of the remaining compartments (Tables 2-4). Besides consisting of miRNAs previously reported by others to be associated with tumor cells, the stromal compartment and tumor microvasculature, as discussed above, the table also provides a glance at miRNAs that have not yet been identified in specific compartments. 


\begin{tabular}{|c|c|c|c|c|c|c|c|c|}
\hline & \multirow{2}{*}{$\begin{array}{l}\text { Microvasculature } \\
\text { microRNAs }\end{array}$} & \multicolumn{4}{|c|}{ Global mean normalized level (dCq) } & \multicolumn{3}{|c|}{ Enrichment factor ( $\left.2^{\mathrm{ddCq}}\right)$} \\
\hline & & $\begin{array}{l}\text { Whole } \\
\text { tumor }\end{array}$ & Tumor (TT) & $\begin{array}{l}\text { Stroma } \\
\text { (ST) }\end{array}$ & TEC & $\begin{array}{l}\text { TT vs } \\
\bar{X}(S T+T E C)\end{array}$ & $\begin{array}{l}\text { ST vs } \\
\bar{X}(T T+T E C)\end{array}$ & $\begin{array}{l}\text { TEC vS } \\
\bar{X}(T T+S T)\end{array}$ \\
\hline 1 & hsa-miR-126-3p & 4.98 & 1.61 & 5.86 & 7.45 & 0.03 & 2.53 & 13.11 \\
\hline 2 & hsa-miR-126-5p & 2.87 & -0.94 & 3.26 & 4.57 & 0.03 & 2.74 & 10.61 \\
\hline 3 & hsa-miR-383 & -12.50 & -7.48 & -7.57 & -4.33 & 0.35 & 0.32 & 9.19 \\
\hline 4 & hsa-miR-451a & 2.86 & -0.74 & 0.78 & 3.21 & 0.15 & 0.73 & 9.14 \\
\hline 5 & hsa-miR-187-3p & -7.59 & -7.48 & -6.98 & -4.15 & 0.26 & 0.45 & 8.47 \\
\hline 6 & hsa-miR-34c-3p & -4.59 & -4.39 & -7.57 & -2.98 & 1.85 & 0.07 & 7.99 \\
\hline 7 & hsa-miR-133b & -3.18 & -5.45 & -2.16 & -0.84 & 0.06 & 1.99 & 7.78 \\
\hline 8 & hsa-miR-503-5p & -5.11 & -5.38 & -5.67 & -2.74 & 0.44 & 0.33 & 6.88 \\
\hline 9 & hsa-miR-486-5p & -2.73 & -5.25 & -4.29 & -2.06 & 0.24 & 0.64 & 6.55 \\
\hline 10 & hsa-miR-373-3p & -12.41 & -6.23 & -7.57 & -4.25 & 0.80 & 0.20 & 6.30 \\
\hline 11 & hsa-miR-144-3p & -4.73 & -5.21 & -5.33 & -2.62 & 0.43 & 0.38 & 6.25 \\
\hline 12 & hsa-miR-133a & -2.87 & -5.15 & -0.58 & -0.72 & 0.04 & 5.11 & 4.44 \\
\hline 13 & hsa-miR-379-5p & -1.74 & -7.48 & -0.46 & -1.82 & 0.01 & 18.34 & 4.43 \\
\hline 14 & hsa-miR-30a-5p & -0.19 & -3.28 & 0.92 & 0.94 & 0.05 & 4.26 & 4.35 \\
\hline 15 & hsa-miR-124-3p & -7.00 & -5.56 & -4.97 & -3.28 & 0.37 & 0.68 & 3.96 \\
\hline 16 & hsa-miR-218-5p & -1.51 & -5.54 & -0.73 & -1.18 & 0.04 & 6.18 & 3.88 \\
\hline 17 & hsa-miR-143-3p & 5.17 & 1.57 & 6.07 & 5.72 & 0.05 & 5.37 & 3.74 \\
\hline 18 & hsa-miR-497-5p & 0.10 & -2.63 & 1.58 & 1.19 & 0.06 & 4.94 & 3.27 \\
\hline 19 & hsa-miR-382-5p & -2.03 & -5.97 & -1.57 & -2.19 & 0.06 & 5.71 & 2.99 \\
\hline 20 & hsa-miR-195-5p & 2.83 & -0.01 & 4.28 & 3.71 & 0.06 & 5.39 & 2.97 \\
\hline
\end{tabular}

\section{- FUTURE PERSPECTIVE}

The current study was initiated to investigate the possibilities and limitations of combining compartmentalization of complex tissues by LMD with miRNA profiling. Such a strategy would allow us to deconstruct the molecular complexity of tissues, including tumor tissues and organ tissues, for application in drug development and biomarker discovery.

The ability to focus miRNA analysis on specific cellular compartments within a heterogeneous, complex tissue made up of several cell types is essential to improve our understanding of the biological role(s) that miRNAs play during various stages of onset and progression of disease [23]. For example, Hu et al. have shown that colon cancer tissue levels of miR-196 are informative of chemoresistance of this type of cancer [24]. Compartment-specific miRNA expression profiles generated in this study show that miR-196a-5p is highly enriched in tumor cells compared with stroma and vasculature (Table 2). Tumor angiogenesis is essential for tumor growth and metastasis. Wang et al. have reviewed the importance of miRNA regulation in tumor angiogenesis, highlighting for example the relevance of miR-126, miR-503, miR-497, miR-218 and miR-195 for the regulation of VEGF, VEGF inducers and VEGF independent factors [25]. Our data confirm that all of these miRNAs are highly expressed in the microvascular compartment compared to stroma and tumor, since all miRNAs rank within the top 20 TEC-enriched miRNAs (Table 4). Thus, the combination of techniques allowing physical separation of such tissues into more homogeneous compartments with sensitive analytical methods is essential to obtain interpretable results from tissue analysis. Using this approach, we were able to demonstrate profound differences in miRNA expression signatures between whole tumor tissue and subsets of cell types in different compartments within the tumor (Figure 4A). The approach presented here is crucial for the discovery of molecular differences between 'healthy and diseased' tissues, as it does not change the status of the cells as is seen with, for example, trypsin treatment, to release them for subsequent molecular analyses. In addition, information about the original location of the cells that we have molecularly mapped is retained, enabling combining of the molecular map with histopathological information. It thus provides an important 


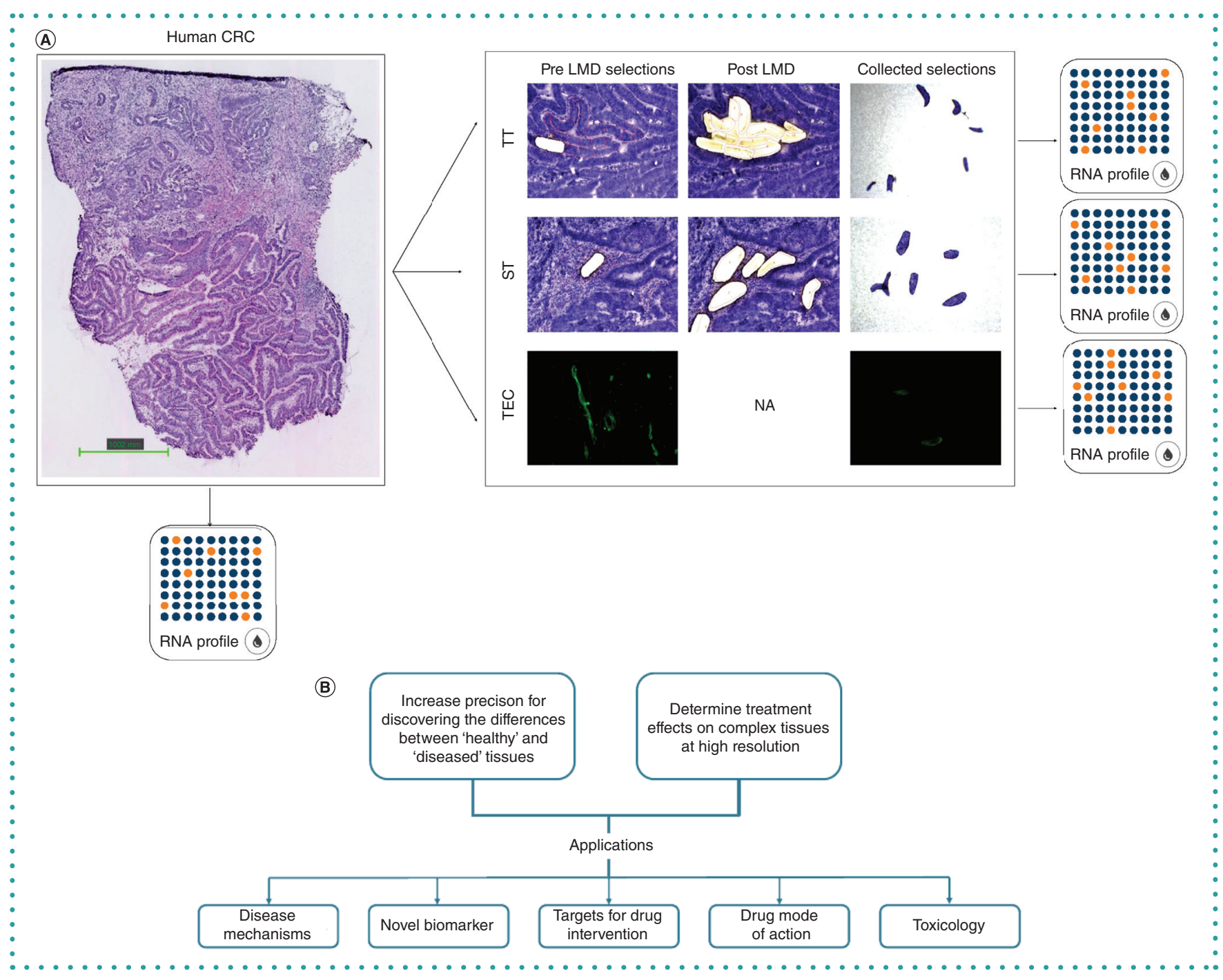

Figure 4. Summary. (A) Malignant (as well as healthy) tissues are mixtures of multiple cell types: tumor cells, stromal cells, microvasculature - mainly endothelial cells - and often other specialized cell types (e.g., tumor-infiltrating lymphocytes). The molecular analysis of whole-tumor tissue samples results in a mixture of signals with unclear origin. Combining LMD to obtain pre-selected cell types with highly sensitive RNA expression analysis is a crucial step toward obtaining interpretable results from complex tissue analysis. (B) This above-depicted strategy enables improvement of identification of molecular signatures that underlie disease processes and responses of multiple cell types to treatment, and as such represents an important novel fundament for drug discovery and drug development.

CRC: Colorectal cancer; LMD: Laser microdissection; NA: Not assessed; ST: Stromal tissue; TEC: Tumor endothelial cell; TT: Tumor tissue.

new combination of technologies to investigate mechanisms of disease, discovery of novel biomarkers and targets for therapeutic intervention, and monitor the effects of drug candidates on specific cell types within the target tissue $[26,27]$ (Figure 4B).

\section{AUTHOR CONTRIBUTIONS}

Individual contributions include the following: MH, GM for the study concept and design; SS for the data collection; PJZ and TK for LMD; MH, SS for miRNA analysis; $\mathrm{MH}$, PJZ, TK, SS, GM for the data analysis and data interpretation; $\mathrm{MH}, \mathrm{GM}$, ES for drafting the manuscript and literature research.

\section{ETHICAL CONDUCT OF RESEARCH}

The authors state that they have obtained appropriate institutional review board approval or have followed the principles outlined in the Declaration of Helsinki for all human or animal experimental investigations. In addition, for investigations involving human subjects, informed consent has been obtained from the participants involved.

\section{ACKNOWLEDGMENTS}

The authors would like to thank Jan Zuidema and Siert Bruins from Vivomicx for their efforts to enable this collaborative research.

\section{FINANCIAL \& COMPETING INTERESTS DISCLOSURE}

Grietje Molema is co-founder and CScientific/ Technology Officer of Vivomicx. Matthias Hackl is co-founder and CEO of TAmiRNA and holds patents related to the use of circulating microRNAs as disease biomarkers. Matthias Hackl, Susanna Skalicky and Elisabeth Schraml are employed by TAmiRNA GmbH. The authors have no other relevant affiliations or financial involvement with any organization or entity with a financial interest in or financial conflict with the subject matter or materials discussed in the manuscript apart from those disclosed. 
- No writing assistance was utilized in the production of this manuscript.

\section{OPEN ACCESS}

This work is licensed under the AttributionNonCommercial-NoDerivatives 4.0 Unported License. To view a copy of this license, visit http://creativecommons.org/licenses/ by-nc-nd/4.0/

\section{REFERENCES}

1. Emmert-Buck MR, Bonner RF, Smith PD et al. Lase capture microdissection. Science 274(5289), 998-100 (1996)

2. Best CJ, Emmert-Buck MR. Molecular profiling of tissue samples using laser capture microdissection. Expert Rev. Mol. Diagn. 1(1), 53-60 (2001).

3. Langenkamp E, Kamps JAAM, Mrug M et al. Innovations in studying in vivo cell behavior and pharmacology in complex tissues - microvascular endothelial cells in

spotlight. Cell. Tissue Res. 354(3), 647-669 (2013).

4. Vannini I, Fanini F, Fabbri M. Emerging roles of microRNAs in cancer. Curr. Opin. Genet. Dev. 48, 128-133 (2018).

5. Ludwig N, Leidinger P, Becker $\mathrm{K}$ et al. Distribution of 作 Res. 44(8), 3865-3877 (2016).

6. Minami K, Uehara T, Morikawa Y, Omura K, Kanki M. miRNA expression atlas in male rat. Sci. Data. 1, 140005 (2014)

7. Leidinger P, Backes $C$, Meder B, Meese E, Keller A. The human miRNA repertoire of different blood compounds. BMC Genomics 1-8 (2014).

8. Schraml E, Hackl M, Grillari J. MicroRNAs and toxicology: a love marriage. Toxicology Rep. 4, 634-636 (2017).

9. Rosenfeld N, Aharonov R, Meiri E et al. MicroRNAs accurately identify cancer tissue origin. Nat. Biotechnol. 26(4), 462-469 (2008)

10. Vandesompele J, Preter KD, Roy NV, Paepe AD, Speleman F. Accurate normalization of real-time quantitative RT-PCR data by geometric averaging of multiple interna control

11. Metsalu T, Vilo J. ClustVis: a web tool for visualizing clustering of multivariate data using Principal Component Analysis and heatmap. Nucleic Acids Res. 43(W1), W566-W570 (2015)

12. Hansen TF, Andersen CL, Nielsen BS et al. Elevated microRNA-126 is associated with high vascular endothelial growth factor receptor 2 expression levels and high microvessel density in colorectal cancer. Oncol. Lett. 2(6), 1101-1106 (2011).

13. Ásgeirsdóttir SA, van Solingen C, Kurniati NF et al. MicroRNA-126 contributes to renal microvascular
heterogeneity of VCAM-1 protein expression in acute heterogeneity of VCAM-1 protein expression in acu
inflammation. Am. J. Physiol.-Ren. Physiol. 302(12), F1630-F1639 (2012).

14. Hirata H, Ueno K, Shahryari V, Tanaka Y, Tabatabai ZL, Hinoda Y. Oncogenic miRNA-182-5p targets Smad4 and RECK in human bladder cancer. PLOS ONE 7(11), 1-8 (2012).

15. Kun-Peng Z, Chun-Lin Z, Xiao-Long M, Lei Z. Fibronectin-1 modulated by the long noncoding RNA OIP5-AS1/ miR-200b-3p axis contributes to doxorubicin resistance of osteosarcoma cells. Cell. Physiol. 234(5), 6927-6939 (2019).

16. Zhu Y, Gu J, Li Y et al. MiR-17-5p enhances pancreatic cancer proliferation by altering cell cycle pro fi les via distuption of RBL2/E2 F4-

17. Harris TA, Yamakuchi M, Ferlito M, Mendell JT, Lowenstein CJ. MicroRNA-126 regulates endothelial expression of vascular cell adhesion molecule 1. Proc. Natl Acad. Sci. USA 105(5), 1516-1521 (2008).

18. Mendelsohn AR, Larrick JW. Rejuvenating muscle stem cell function: Rejuvenation Res. restoring quiescence and overcoming senescence. 19(2), 182-186 (2016).

19. Qu M, Pan J, Shi X, Zhang Z, Tang Y, Yang G. MicroRNA-126 is a prospective target for vascular disease. Neuroimmunol. Neuroinflammation. 5(10), 1-7 (2018).

20. Calvano J, Achanzar W, Murphy B et al. Evaluation of microRNAs-208 and $133 \mathrm{a} / \mathrm{b}$ as differential biomarke Toxicol. Appl. Pharmacol. 312, 53-60 (2015).
21. Chen C, Zhao Z, Liu YU, Mu D. microRNA-99a is downregulated and promotes proliferation, migration and cells. Oncol. Lett. 9(3), 1128-1134 (2015).

22. Milosevic J, Pandit K, Magister M et al. Profibrotic role of miR-154 in pulmonary fibrosis. Am. J. Respir. Cell Mol. Biol. Vol. 32(6), 879-887 (2012).

23. Simões $A$, Chen $Z$, Zhao $Y$ et al. Laser capture microdissection of epithelium from a wound healing model for microRNA analysis. In: MicroRNA Protocols. Humana Press, NY, USA, 1733, 225-237 (2018).

24. Hu J, Xu Y, Cai S. Specific microRNAs as novel biomarkers for combination chemotherapy resistance 20, 95 (2015).

25. Wang $Y$, Wang $L$, Chen $C$, Chu $X$. New insights into the egulatory role of microRNA in tumor angiogenesis and clinical implications. Mol. Cancer. 17(1), 22 (2018).

26. Asgeirsdottir SA, Kamps JAAM, Bakker HI et al. Site-specific inhibition of glomerulonephritis progression by targeted delivery of dexamethasone to glomerular endothelium. Mol. Pharmacol. 72(1), 121-131 (2007).

27. Kowalski PS, Zwiers PJ, Morselt HWM et al. Anti-VCAM-1 SAINT-O-Somes enable endothelial-specific delivery of siRNA and downregulation of inflammatory Release. 176, 64-75 (2014) Leidinger P. Backes C Meder $B$ Meese $E$, Keller A. The human miRNA repertoire of different blood compounds. BMC Genomics 1-8 (2014). 